\title{
Structure of human mitochondrial RNA polymerase
}

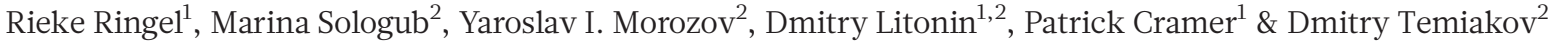

Transcription of the mitochondrial genome is performed by a single-subunit RNA polymerase (mtRNAP) that is distantly related to the RNAP of bacteriophage T7, the pol I family of DNA polymerases, and single-subunit RNAPs from chloroplasts ${ }^{1-4}$. Whereas T7 RNAP can initiate transcription by itself, mtRNAP requires the factors TFAM and TFB2M for binding and melting promoter $\mathrm{DNA}^{5-7}$. TFAM is an abundant protein that binds and bends promoter DNA 15-40 base pairs upstream of the transcription start site, and stimulates the recruitment of mtRNAP and TFB2M to the promoter ${ }^{8,9}$. TFB2M assists mtRNAP in promoter melting and reaches the active site of mtRNAP to interact with the first base pair of the RNA-DNA hybrid ${ }^{10}$. Here we report the $\mathrm{X}$-ray structure of human mtRNAP at $2.5 \AA$ resolution, which reveals a T7-like catalytic carboxy-terminal domain, an amino-terminal domain that remotely resembles the $\mathrm{T} 7$ promoter-binding domain, a novel pentatricopeptide repeat domain, and a flexible $\mathrm{N}$-terminal extension. The pentatricopeptide repeat domain sequesters an AT-rich recognition loop, which binds promoter DNA in T7 RNAP, probably explaining the need for TFAM during promoter binding. Consistent with this, substitution of a conserved arginine residue in the AT-rich recognition loop, or release of this loop by deletion of the $\mathrm{N}$-terminal part of $\mathrm{mtRNAP}$, had no effect on transcription. The fingers domain and the intercalating hairpin, which melts DNA in phage RNAPs, are repositioned, explaining the need for TFB2M during promoter melting. Our results provide a new venue for the mechanistic analysis of mitochondrial transcription. They also indicate how an early phage-like mtRNAP lost functions in promoter binding and melting, which were provided by initiation factors in trans during evolution, to enable mitochondrial gene regulation and the adaptation of mitochondrial function to changes in the environment.

We crystallized a fully functional variant of a recombinant human mtRNAP (residues 105-1230) that requires the presence of both TFAM and TFB2M for efficient transcription initiation on a doublestranded promoter DNA (Supplementary Fig. 1). The structure was determined at $2.5 \AA$ resolution by molecular replacement with the use of a truncated T7 RNAP structure as a search model ${ }^{2}$, and was refined to a free $R$-factor of 0.23 (Methods, and Supplementary Table 1).

The mtRNAP structure has the shape of a right hand with palm, fingers and thumb subdomains, characteristic of the pol A family of a

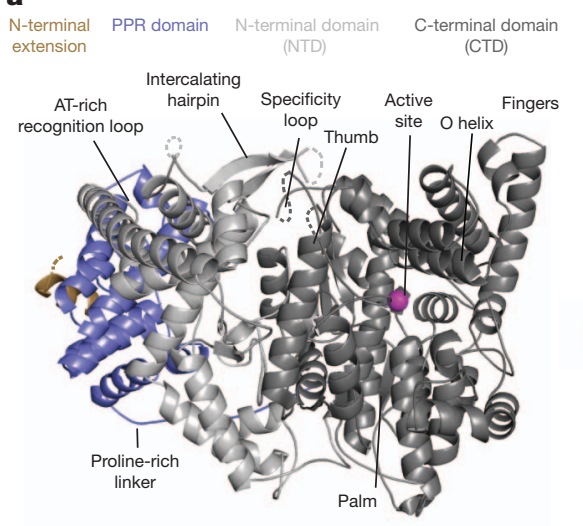

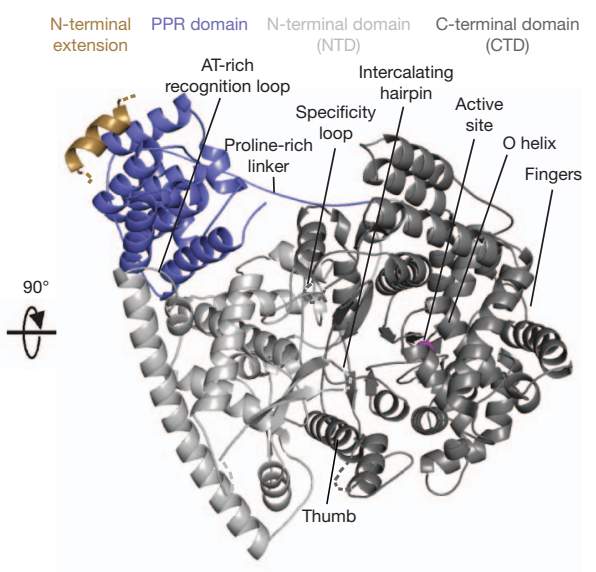

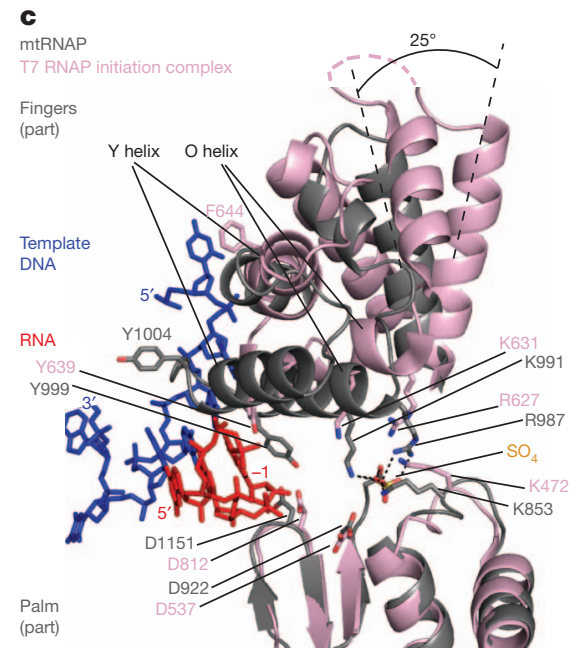

C
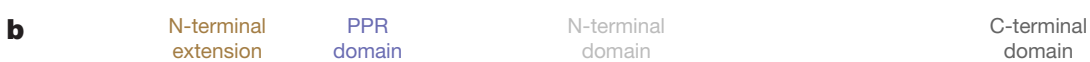

domain Specificity

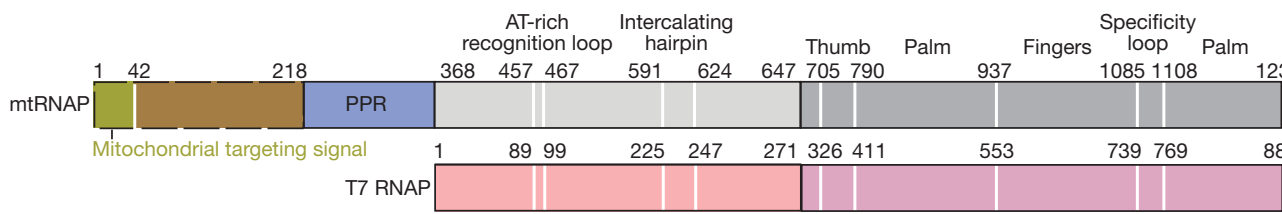

Figure 1 Crystal structure of human mtRNAP. a, Two views of a ribbon model with the major domains and structural elements indicated. The CTD that is conserved in all single-stranded RNAPs is in dark grey, the NTD in silver, the PPR domain in blue, and the $\mathrm{N}$-terminal extension helix in sand. The active site is indicated by a magenta sphere for a modelled catalytic metal ion. b, Schematic comparison of mtRNAP with T7 (PDB 1QLN) RNAP. Prominent structural elements are indicated. mtRNAP-specific residues 1-368 include the mitochondrial targeting signal, the $\mathrm{N}$-terminal extension and the PPR domain.
Regions in mtRNAP that are not visible in the crystal structure include residues 592-602, 736-769 and 1086-1105. c, Superimposition of the active-centre regions of mtRNAP (grey) and the T7 RNAP initiation complex (PDB 1QLN, light pink) reveals a clenched conformation due to a roughly $25^{\circ}$ rotation of the fingers subdomain around the O-helix axis. Template DNA is in blue, RNA in red. Conserved catalytic residues, residues implicated in NTP binding, and a sulphate ion are shown with sticks.

${ }^{1}$ Gene Center and Department of Biochemistry, Center for Integrated Protein Science Munich (CIPSM), Ludwig-Maximilians-Universität München, Feodor-Lynen-Strasse 25, 81377 Munich, Germany. ${ }^{2}$ Department of Cell Biology, University of Medicine and Dentistry of New Jersey, School of Osteopathic Medicine, 2 Medical Center Dr, Stratford, New Jersey 08084, USA. 
nucleotidyltransferases (Fig. 1). We use here the canonical polymerase domain nomenclature and the previously defined names for functional elements in T7 RNAP ${ }^{2,11}$, even though these have functional implications that are not borne out in mtRNAP. The highly conserved palm in the C-terminal domain (CTD) superimposes well on the corresponding palm in T7 RNAP (root mean squared deviation 1.0 ̊, 121 backbone atoms). The marked conservation of the active centre, including the Ohelix, suggests that the mechanisms of substrate binding and selection are conserved between mtRNAP and T7 RNAP ${ }^{12,13}$. The O helix binds a sulphate ion with its residues R987, K991 and K853 (Fig. 1c) near the position of the phosphate groups of an incoming nucleoside triphosphate (NTP) in the T7 RNAP elongation complex $^{12,13}$. A portion of the thumb (residues 736-769) and the flexible specificity loop (residues 1086-1105) are not visible in the structure, but adjacent elements occupy almost identical positions in T7 RNAP, indicating that these elements have similar orientations and functions.
The most notable difference between the CTD of T7 RNAP and mtRNAP is the position of the fingers subdomain, which is rotated as a rigid body by roughly $25^{\circ}$ approximately around the axis of the $\mathrm{O}$ helix, accompanied by a tilting of the $\mathrm{O}$ and $\mathrm{Y}$ helices (Fig. 1c). These movements result in a $15 \AA$ translation of the $\mathrm{N}$-terminal part of the Y helix towards the promoter-binding domain (PBD). The observed position of the fingers domain is distinct from the previously observed positions in the 'closed' elongation-complex structure of T7 RNAP, in which the O helix is positioned to deliver the NTP into the active site ${ }^{12}$ (Fig. 1c). We refer to this novel RNAP conformation as 'clenched'. The clenched conformation is unlikely to occur during the nucleotide addition cycle, because the entrance to the active site may be occluded by the conserved Y-helix residue Y1004, which was implicated in downstream duplex melting ${ }^{14,15}$ and is partly mobile in our structure. It is unclear whether the clenched conformation is functional or is a result of crystal packing.

a

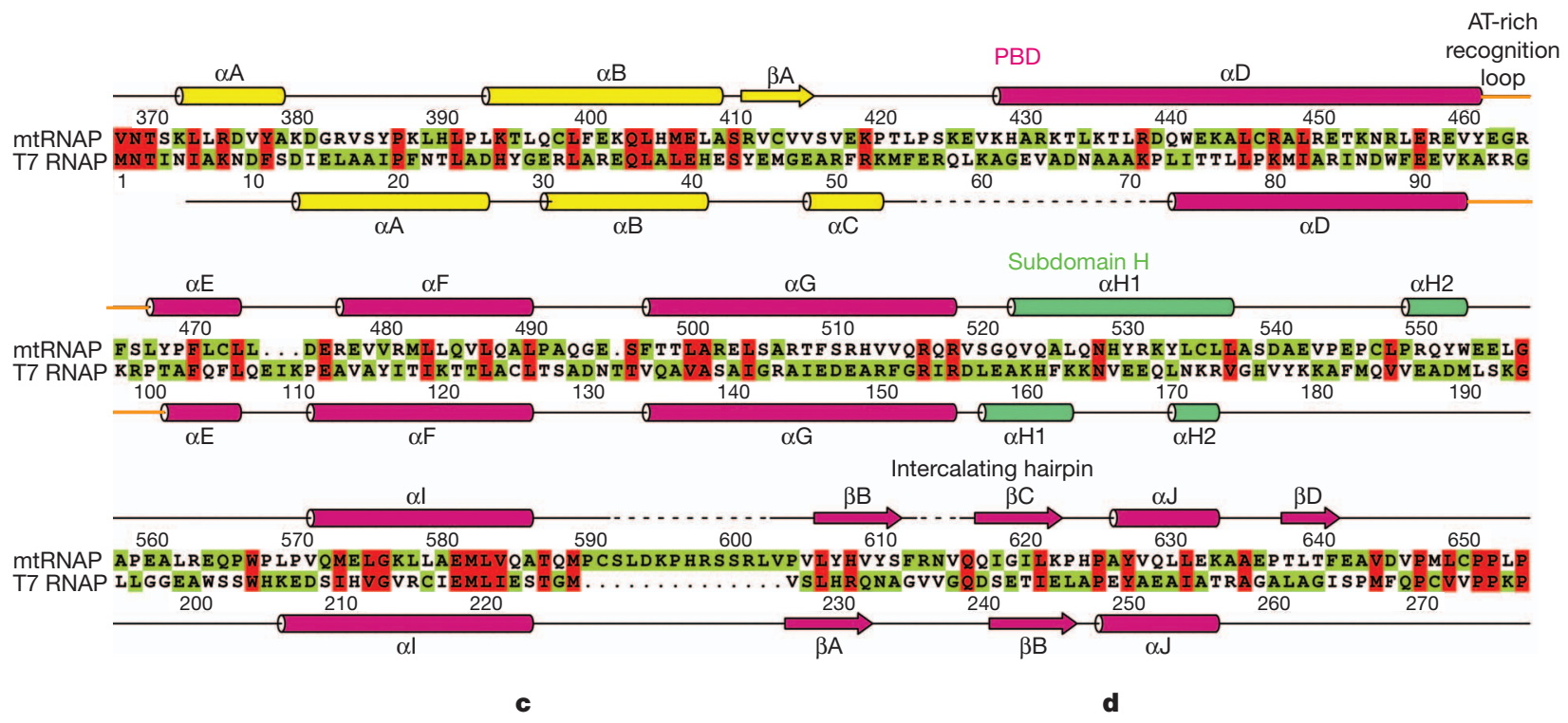

b

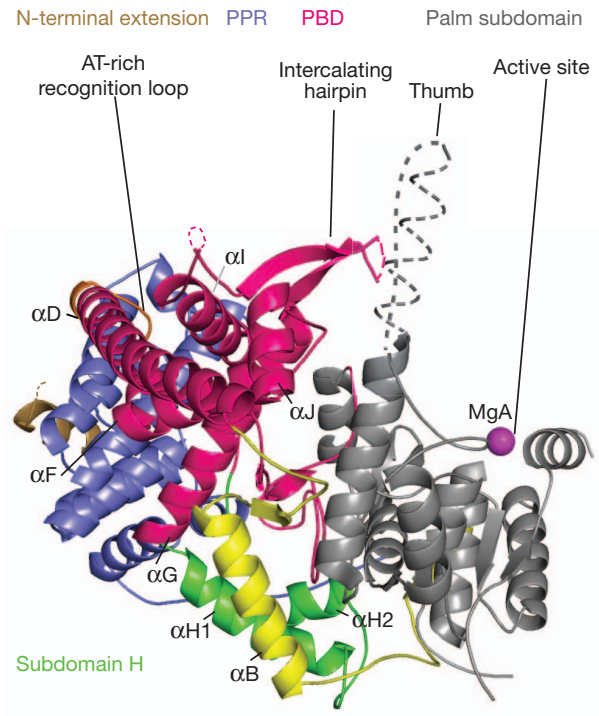

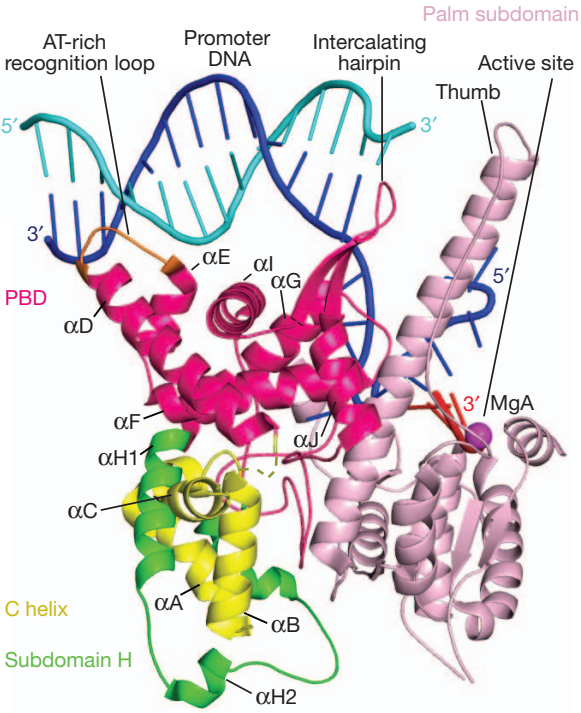

d

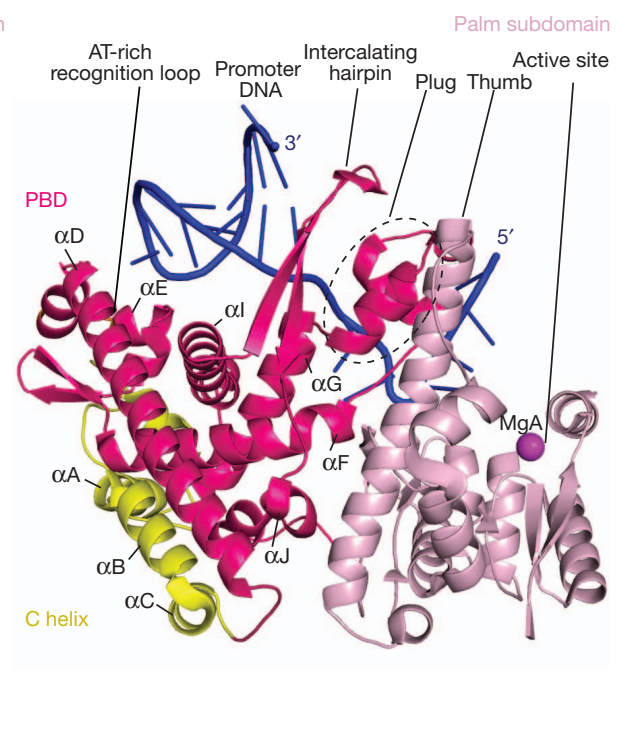

Figure $2 \mid$ Variation in RNAP N-terminal domains. a, Sequence alignment and structural conservation of human mtRNAP and T7 RNAP (PDB 1QLN). Secondary-structure elements are indicated above and below the sequences for mtRNAP (residues 368-654) and T7 RNAP, respectively (cylinders, $\alpha$-helices; arrows, $\beta$-strands; lines, loops). The PBD is in pink, the $\mathrm{C}$ helix in yellow, and subdomain $\mathrm{H}$ in green. The AT-rich recognition loop (orange) and the intercalating hairpin are indicated. Identical residues are highlighted in red. Dashed lines indicate regions that are not visible in the mtRNAP structure. b-d, Comparison of the NTDs of mtRNAP (b) with initiation complexes of T7 RNAP (PDB 1QLN) (c) and N4 RNAP (PDB 3Q24) (d). Structures are aligned with respect to their conserved palm subdomains. Colour code and residue borders are as in a. RNA is in red, template DNA in blue, and a non-template strand in cyan. Of the CTD (grey or light pink) only the palm subdomain (residues 790-831, 911-937 and 1125-1178) and the thumb (residues 677790) are shown. 
The large N-terminal region of mtRNAP (residues 1-647; Fig. 1b) shares no sequence homology with T7 RNAP. Nevertheless, the C-terminal part of this region (residues 369-647) is structurally similar to the N-terminal domain of phage RNAPs, and we therefore refer to it as the N-terminal domain (NTD). In particular, the six-helix bundle of the PBD in phage RNAPs, which includes helices D, E, F, G, I and J, has a counterpart in mtRNAP (Fig. 2). However, there are tangible changes in the orientation of the PBD helices between mtRNAP and T7 RNAP (root mean squared deviation $9 \AA$; 109 backbone atoms). In comparison with T7 RNAP, the N-terminal part of the PBD (residues $425-519$ ) is rotated by $32^{\circ}$, whereas the C-terminal part (residues $567-654$ ) is rotated by $24^{\circ}$ in mtRNAP.

The structure further reveals the loops corresponding to the intercalating hairpin and the AT-rich recognition loop of T7 RNAP. The hairpin connects the conserved helices I and J and has a 14-residue insertion that is partly visible in the mtRNAP structure (Fig. 2a). The loop that connects helices $\mathrm{D}$ and $\mathrm{E}$ of the PBD corresponds to the ATrich recognition loop but is shorter, whereas the $\mathrm{D}$ helix is extended by comparison with T7 RNAP (Fig. 2b, c). Another significant difference in the NTD of mtRNAP is the alternative fold of the region at the position of subdomain $\mathrm{H}$ in T7 RNAP. It is likely that this region of the mtRNAP NTD undergoes refolding during the transition from initiation to elongation, to form a part of an RNA exit pore, as described for T7 RNAP ${ }^{11,14,15}$. It remains to be seen whether the observed conformation is specific for mtRNAP or whether it also occurs in T7 RNAP but has not yet been observed. In phage N4 RNAP, which is distantly related to T7 RNAP, subdomain $\mathrm{H}$ is absent (Fig. 2d).

The NTD of mtRNAP is connected to a unique helical domain (residues 218-368) through an extended, nearly linear, proline-rich linker (Figs 1 and 3 ). This domain consists of nine $\alpha$-helices, of which four comprise two pentatricopeptide repeat (PPR) motifs found in plant and mitochondrial proteins ${ }^{16-18}$. We refer to this domain as the PPR domain. PPR-motif-containing proteins are apparently involved in RNA editing and processing ${ }^{19,20}$, but their structures are unknown. Our structure of the PPR domain shows that the conserved residues of the PPR motif form the hydrophobic core of a helix-turnhelix fold and the interface between antiparallel $\alpha$-helices $\left(\alpha \mathrm{D}^{\prime}\right.$ and $\alpha \mathrm{E}^{\prime}, \alpha \mathrm{E}^{\prime}$ and $\alpha \mathrm{F}^{\prime}, \alpha \mathrm{F}^{\prime}$ and $\left.\alpha \mathrm{G}^{\prime}\right)$ and parallel helices $\left(\alpha \mathrm{E}^{\prime}\right.$ and $\left.\alpha \mathrm{G}^{\prime}\right)$
(Fig. 3a, b). The PPR domain interacts tightly with the NTD. Helices $\alpha \mathrm{A}^{\prime}$ and $\alpha \mathrm{B}^{\prime}$ form a large hydrophobic interface (about $950 \AA^{2}$ ) that includes the tip of the AT-rich recognition loop and its flanking helices $\alpha \mathrm{E}$ and $\alpha \mathrm{D}$ (Fig. 3c). In addition, a salt bridge between R225 and E574 and numerous hydrogen bonds stabilize the PPR-NTD interface (Fig. 3c).

The region located $\mathrm{N}$-terminal of the PPR domain, called here the $\mathrm{N}$-terminal extension, is mostly mobile, except for a single unassigned $\alpha$-helix that docks to a hydrophobic pocket formed by $\alpha \mathrm{F}^{\prime}$ and $\alpha \mathrm{G}^{\prime}$ of the PPR domain (Fig. 3b). The N-terminal extension of human mtRNAP is required for function, because deletion of the N-terminal 200 residues results in a truncated polymerase that is catalytically active but unable to initiate promoter-directed transcription (Supplementary Fig. 1).

Footprinting experiments suggested that during transcription initiation mtRNAP (in complex with TFB2M) and T7 RNAP occupy 14 and 17 base pairs, respectively, of upstream promoter $\mathrm{DNA}^{7,10}$ (Fig. 4a). The rest of the upstream promoter region in mammalian mtDNA $(-35$ to -15$)$ is covered by TFAM $^{7}$, suggesting that mtRNAP and T7 RNAP use different modes of promoter binding. To explore this we modelled the putative trajectory of upstream promoter DNA in mtRNAP by superimposing the palm subdomains of mtRNAP and the T7 RNAP initiation complex ${ }^{21}$ (Fig. 4b). In the resulting model, which remains tentative because DNA binding can cause structural changes, upstream promoter DNA runs along the NTD, and the template single strand descends into the mtRNAP active site (Figs 1c and 4b). The tip of the specificity loop may reach into the major groove of promoter DNA and read the sequence between -6 and -11 base pairs while its base may interact with the phosphodiester backbone of a single-stranded DNA, essentially as in phage RNAPs ${ }^{21,22}$. This is consistent with a functional analysis of point mutations in the specificity loop of yeast mtRNAP ${ }^{23}$.

Modelling also reveals structural differences in mtRNAP, relative to T7 RNAP, that may render mtRNAP reliant on the initiation factor TFB2M and responsive to activation by TFAM. In particular, the AT-rich recognition loop has distinct structures and functions in the two systems. In comparison with T7 RNAP, helix D is rotated away from the minor groove of DNA by $45^{\circ}$ and the tip of the ATrich recognition loop is shifted by $22 \AA$ (Fig. 4c) and sequestered by

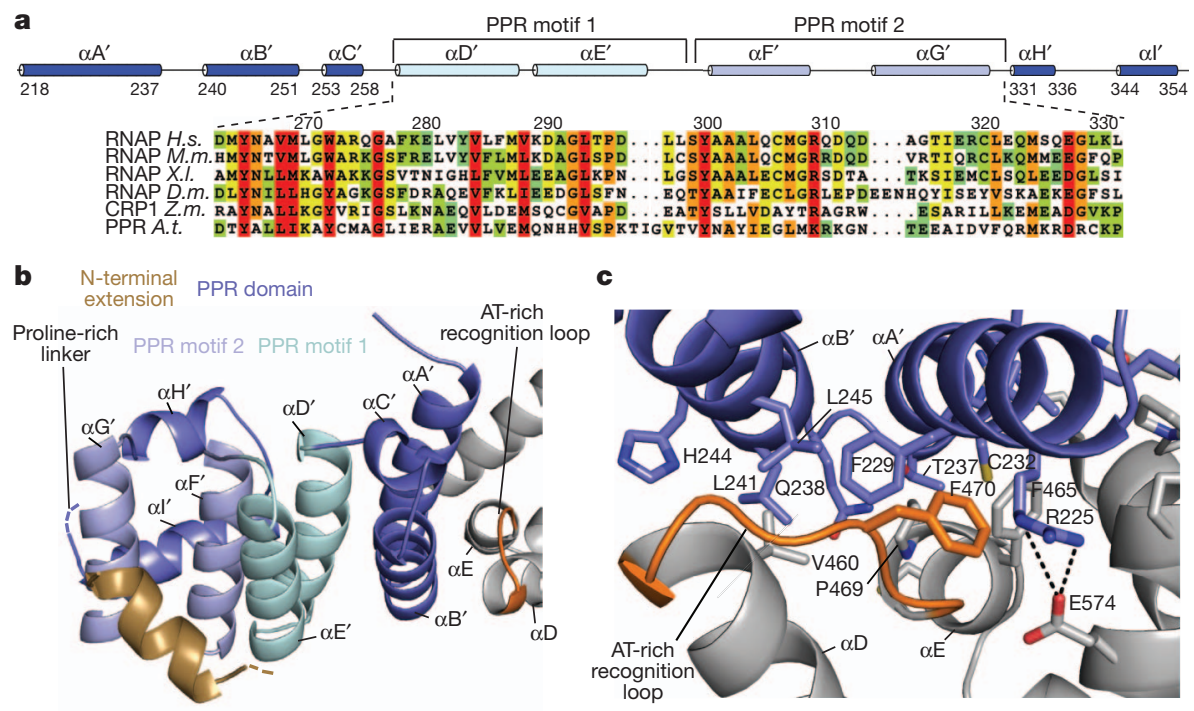

Figure 3 PPR domain. a, Sequence alignment of human mtRNAP PPR domain, including PPR motifs 1 and 2, with predicted PPR motifs in mtRNAPs of different species (lanes 2-4), and chloroplast-targeting proteins of plants (lines 5,6). Abbreviations: H.s., Homo sapiens; M.m., Mus musculus; X.l., Xenopus laevis; D.m., Drosophila melanogaster; Z.m., Zea mays; A.t., Arabidopsis thaliana. Residues that are identical, highly conserved, conserved, and distinct, are highlighted in red, orange, yellow, and green, respectively. b, Structure of PPR domain (blue) with PPR motifs 1 (light cyan) and 2 (light blue). Each PPR motif shows a helix-turn-helix fold. Helices $\alpha \mathrm{A}^{\prime}$ and $\alpha \mathrm{B}^{\prime}$ are adjacent to the NTD (silver) and interact with the AT-rich recognition loop (orange). PPR motif 1 interacts with a helix in the $\mathrm{N}$-terminal extension (sand). c, Interface between PPR domain and NTD. Colours are as in a. Interface residues are shown in stick representation. 
a

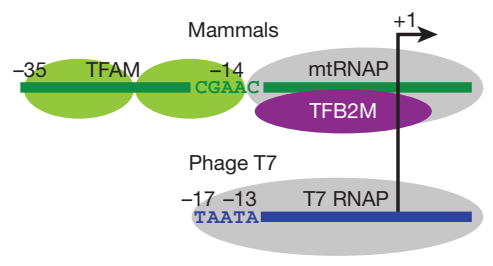

C

N-terminal extension PPR domain PBD

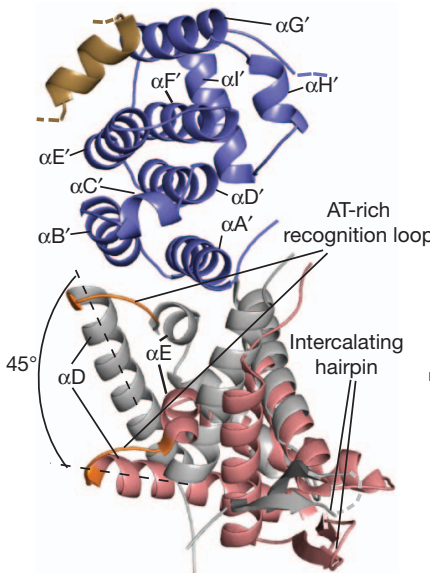

b
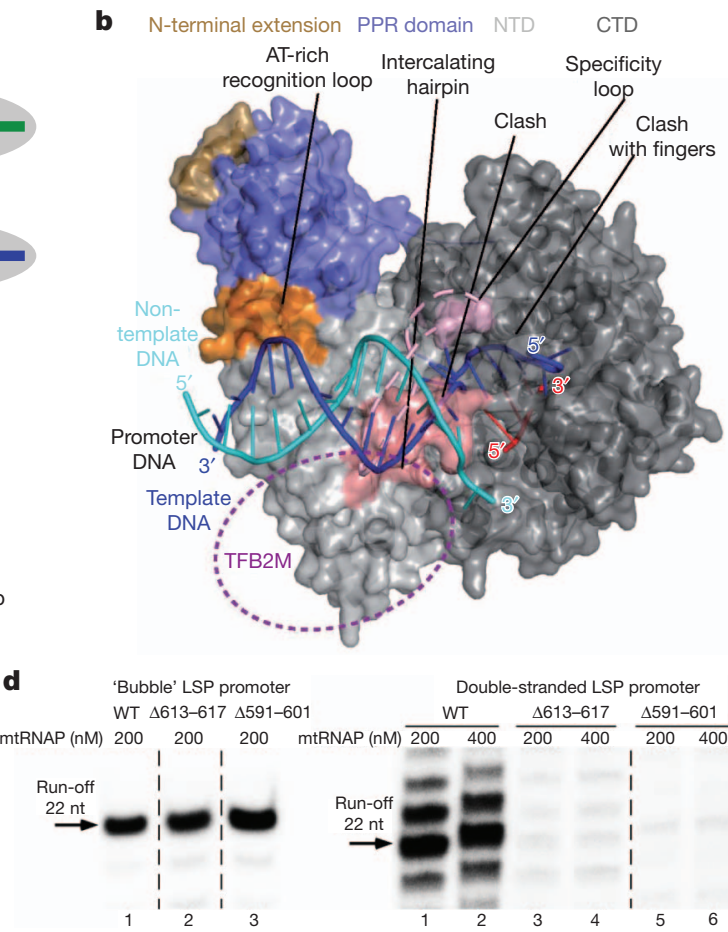

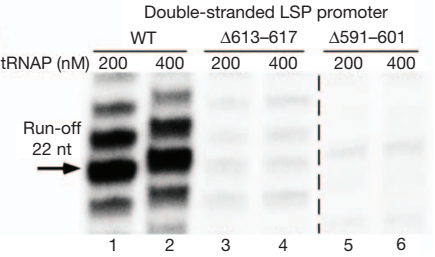

Figure $4 \mid$ Promoter binding and melting by mtRNAP. a, Differences in topology of the initiation complexes of T7 and mammalian mitochondrial transcription machineries, based on foot-printing data and functional assays. b, Modelled location of upstream promoter DNA on mtRNAP, based on a superimposition with the T7 RNAP initiation complex (PDB 1QLN). mtRNAP is depicted as a molecular surface. Loops in mtRNAP that interact with DNA in the T7 system are indicated. The portion of the specificity loop not visible in the structure is modelled based on the corresponding T7 loop (dashed line). The AT-rich recognition loop is buried by the PPR domain (blue) and not seen. The intercalating hairpin (rose) is shifted by $7 \AA$ compared with the T7 RNAP initiation complex, and clashes with the template DNA strand (blue). A clash is also observed between the fingers subdomain and the +1 template base. The presumed binding site of TFB2M is indicated by a dash oval. c, Relative

extensive interactions with the PPR domain (Fig. 3c). Consistent with this, a mtRNAP variant that lacks the PPR domain, and should contain a free AT-rich recognition loop, cannot initiate transcription (Supplementary Fig. 1). In addition, the positively charged residues important for promoter interactions by T7 RNAP are not conserved in the mtRNAP loop, and mutations R458A/R464A have no effect on transcription activity (Supplementary Fig. 2). These results indicate that human mtRNAP does not use its AT-rich recognition loop for promoter binding, explaining why it depends on transcription factors for initiation. The AT-rich recognition loop also has a different structure and function in RNAP of phage N4, where it recognizes a hairpin $\operatorname{promoter}^{22}$ (Fig. 2d).

mtRNAP shows two additional structural differences relative to T7 RNAP that explain why it can not melt DNA and requires TFB2M for promoter melting. First, the position of the intercalating hairpin is not compatible with promoter melting as observed in T7 RNAP (Fig. 2b, c). The tip of the loop is translated $7 \AA$ away from its position in T7 and clashes with the template strand of DNA (Fig. 4b). Second, a $25^{\circ}$ rotation of the fingers domain towards the NTD (Fig. 1c) blocks access of the single-stranded DNA to the active site.

Two mechanisms of promoter melting may be considered. First, binding of TFB2M may reposition the intercalating hairpin so that it can function as in $\mathrm{T} 7$ and N4 RNAP ${ }^{21,22}$. Second, DNA melting may be achieved not by the intercalating hairpin but by an unidentified structural element in TFB2M. To investigate this we constructed variants of mtRNAP lacking either five residues at the tip of the intercalating hairpin $(\Delta 613-617)$ or lacking a preceding insertion $(\Delta 591-601)$. orientation of the PBDs of mtRNAP (silver) and T7 RNAP initiation complex (PDB 1QLN, salmon) based on homology modelling of the corresponding palm subdomain. The AT-rich recognition loop (orange) is shifted and rotated by $45^{\circ}$. d, An intercalating hairpin region is required for promoter melting in mtRNAP. Transcription run-off assays with wild-type (WT) and mutant RNAPs were performed with bubble (left panel) and double-stranded (right panel) light-strand promoter templates for $30 \mathrm{~min}$ at $35^{\circ} \mathrm{C}$. Transcription factors TFAM $(50 \mathrm{nM})$ and TFB2M $(150 \mathrm{nM})$ were added to reactions that involved double-stranded template (right panel), whereas transcription with the bubble template was factor-independent (left panel). Reaction products were resolved with $20 \%$ PAGE containing $6 \mathrm{M}$ urea, and analysed with a PhosphorImager. nt, nucleotides.

These mutant polymerases had an activity similar to that of the wild-type enzyme on a pre-melted 'bubble' light-strand promoter template (Fig. $4 \mathrm{~d}$ ) but were essentially inactive on a double-stranded lightstrand promoter template, suggesting a role of the intercalating hairpin in DNA melting (Fig. 4d). To explore this further, we built a model of a mitochondrial transcription initiation complex, which takes available structural $^{24}$, biochemical and genetic data ${ }^{10,25-27}$ into account (Supplementary Fig. 3). The model remains tentative but indicates that TFB2M may insert between the intercalating hairpin and thumb subdomain, to reposition the intercalating hairpin and the fingers, such that they function in promoter melting (Supplementary Fig. 4). TFB2M may also reach near the active site to stabilize an early transcribing complex by binding the template strand and incoming NTP as suggested $^{10}$.

\section{METHODS SUMMARY}

Human mtRNAP, TFB2M and TFAM were expressed and purified as described ${ }^{10}$ The coding sequences of human mtRNAP N-terminal deletion mutants $\Delta 104$ (residues 105-1230), $\Delta 200(201-1230)$ and $\Delta 368$ (369-1230) were amplified by PCR and cloned into vector pProEx $(\mathrm{Hb})$ (Invitrogen), to express N-terminally hexahistidine-tagged proteins. Deletion mutants $\Delta 613-617$ and $\Delta 591-601 \mathrm{GG}$ and substitution mutant R458A/R464A were obtained by site-directed mutagenesis (Quick-change; Stratagene). Transcription run-off reactions were performed with synthetic DNA templates as described ${ }^{10}$ (Supplementary Fig. 1). For crystallization, $\Delta 104 \mathrm{mtRNAP}$ was treated in situ with $\operatorname{ArgC}$ protease $(1: 1,000 \mathrm{w} / \mathrm{w})$ for $1-2 \mathrm{~h}$ at $23{ }^{\circ} \mathrm{C}$. Initial crystals were obtained in hanging drops over $12 \%$ poly(ethylene glycol) (PEG) 4000, 10\% glycerol, $30 \mathrm{mM} \mathrm{MES,} 60 \mathrm{mM}\left(\mathrm{NH}_{4}\right)_{2} \mathrm{SO}_{4}, 40 \mathrm{mM}$ Tris$\mathrm{HCl} \mathrm{pH} \mathrm{8.0,20} \mathrm{mM} \mathrm{dithiothreitol} \mathrm{at} 20^{\circ} \mathrm{C}$, and used for micro-seeding, which 
produced rod-shaped crystals of up to $0.3 \times 0.1 \times 0.1 \mathrm{~mm}^{3}$ in size. Diffraction data were collected in $0.5^{\circ}$ increments at $100 \mathrm{~K}$ on a MarCCD detector at the Swiss Light Source in Villigen, Switzerland (Supplementary Table 1). Data were integrated with MOSFLM $^{28}$ and scaled with SCALA ${ }^{29}$. The structure was solved by molecular replacement using the T7 RNAP structure ${ }^{2}$ (PDB 1QLN) truncated to polyalanine and reduced to residues $246-354,392-568,691-741$ and 766-883. The replacement solution was subjected to rigid-body refinement with Refmac-5 (ref. 30). The model was iteratively built with COOT and refined with autoBuster (Global Phasing) to a final free $R$-factor of $23 \%$. The refined structure includes residues 218-591, 603-611, 616-735, 770-1085 and 1109-1230. Residues 326-360 and the helix in the N-terminal extension were built as polyalanine because of poor sidechain density. Figures were prepared with Pymol (Schrödinger).

Received 9 May; accepted 10 August 2011.

Published online 25 September 2011.

1. Masters, B. S., Stohl, L. L. \& Clayton, D. A. Yeast mitochondrial RNA polymerase is homologous to those encoded by bacteriophages T3 and T7. Cell 51, 89-99 (1987).

2. Cheetham, G. M. \& Steitz, T. A. Structure of a transcribing T7 RNA polymerase initiation complex. Science 286, 2305-2309 (1999).

3. Gaspari, M. Larsson, N. G. \& Gustafsson, C. M. The transcription machinery in mammalian mitochondria. Biochim. Biophys. Acta 1659, 148-152 (2004).

4. Kohlstaedt, L. A., Wang, J., Friedman, J. M., Rice, P. A. \& Steitz, T. A. Crystal structure at 3.5 A resolution of HIV-1 reverse transcriptase complexed with an inhibitor. Science 256, 1783-1790 (1992).

5. Litonin, D. et al. Human mitochondrial transcription revisited: only TFAM and TFB2M are required for transcription of the mitochondrial genes in vitro. J. Biol. Chem. 285, 18129-18133 (2010).

6. Falkenberg, M. et al. Mitochondrial transcription factors B1 and B2 activate transcription of human mtDNA. Nature Genet. 31, 289-294 (2002)

7. Gaspari, M., Falkenberg, M., Larsson, N. G. \& Gustafsson, C. M. The mitochondrial RNA polymerase contributes critically to promoter specificity in mammalian cells. EMBO J. 23, 4606-4614 (2004).

8. Ekstrand, M. I. et al. Mitochondrial transcription factor A regulates mtDNA copy number in mammals. Hum. Mol. Genet. 13, 935-944 (2004).

9. Dairaghi, D. J., Shadel, G. S. \& Clayton, D. A. Human mitochondrial transcription factor $A$ and promoter spacing integrity are required for transcription initiation. Biochim. Biophys. Acta 1271, 127-134 (1995).

10. Sologub, M., Litonin, D., Anikin, M., Mustaev, A. \& Temiakov, D. TFB2 is a transient component of the catalytic site of the human mitochondrial RNA polymerase. Cell 139, 934-944 (2009).

11. Durniak, K. J., Bailey, S. \& Steitz, T. A. The structure of a transcribing T7 RNA polymerase in transition from initiation to elongation. Science 322, 553-557 (2008).

12. Yin, Y. W. \& Steitz, T. A. The structural mechanism of translocation and helicase activity in T7 RNA polymerase. Cell 116, 393-404 (2004).

13. Temiakov, D. et al. Structural basis for substrate selection by T7 RNA polymerase Cell 116, 381-391 (2004)

14. Tahirov, T. H. et al. Structure of a T7 RNA polymerase elongation complex at $2.9 \AA$ resolution. Nature 420, 43-50 (2002).

15. Yin, Y. W. \& Steitz, T. A. Structural basis for the transition from initiation to elongation transcription in T7 RNA polymerase. Science 298, 1387-1395 (2002).

16. Rodeheffer, M. S., Boone, B. E., Bryan, A. C. \& Shadel, G. S. Nam1p, a protein involved in RNA processing and translation, is coupled to transcription through an interaction with yeast mitochondrial RNA polymerase. J. Biol. Chem. 276, 8616-8622 (2001)

17. Small, I. D. \& Peeters, N. The PPR motif-a TPR-related motif prevalent in plant organellar proteins. Trends Biochem. Sci. 25, 46-47 (2000).

18. Lightowlers, R. N. \& Chrzanowska-Lightowlers, Z. M. PPR (pentatricopeptide repeat) proteins in mammals: important aids to mitochondrial gene expression. Biochem. J. 416, e5-e6 (2008).
19. Hammani, K. et al. The pentatricopeptide repeat protein OTP87 is essential for RNA editing of nad 7 and atp 1 transcripts in Arabidopsis mitochondria. J. Biol. Chem. 286, 21361-21371 (2011)

20. Aphasizheva, I., Maslov, D., Wang, X., Huang, L. \& Aphasizhev, R. Pentatricopeptide repeat proteins stimulate $m R N A$ adenylation/uridylation to activate mitochondrial translation in trypanosomes. Mol. Cell 42, 106-117 (2011).

21. Cheetham, G. M., Jeruzalmi, D. \& Steitz, T. A. Structural basis for initiation of transcription from an RNA polymerase-promoter complex. Nature 399, 80-83 (1999).

22. Gleghorn, M. L., Davydova, E. K., Rothman-Denes, L. B. \& Murakami, K. S. Structural basis for DNA-hairpin promoter recognition by the bacteriophage N4 virion RNA polymerase. Mol. Cell 32, 707-717 (2008)

23. Nayak, D., Guo, Q. \& Sousa, R. A promoter recognition mechanism common to yeast mitochondrial and phage T7 RNA polymerases. J. Biol. Chem. 284, 13641-13647 (2009)

24. Schubot, F. D. et al. Crystal structure of the transcription factor sc-mtTFB offers insights into mitochondrial transcription. Protein Sci. 10, 1980-1988 (2001).

25. Mangus, D. A., Jang, S. H. \& Jaehning, J. A. Release of the yeast mitochondrial RNA polymerase specificity factor from transcription complexes. J. Biol. Chem. 269 26568-26574 (1994).

26. Cliften, P. F., Park, J.Y., Davis, B. P., Jang, S. H. \& Jaehning, J. A. Identification of three regions essential for interaction between a sigma-like factor and core RNA polymerase. Genes Dev. 11, 2897-2909 (1997).

27. Savkina, M., Temiakov, D., McAllister, W. T. \& Anikin, M. Multiple functions of yeast mitochondrial transcription factor Mtf1p during initiation. J. Biol. Chem. 285, 3957-3964 (2010)

28. Leslie, A. G. W. Joint CCP4 and ESF-EACMB Newsletter Protein Crystallogr. No. 26 (Daresbury Laboratory, 1992)

29. Evans, P. Scaling and assessment of data quality. Acta Crystallogr. D Biol. Crystallogr. $62,72-82(2006)$

30. Murshudov, G. N., Vagin, A. A. \& Dodson, E. J. Refinement of macromolecular structures by the maximum-likelihood method. Acta Crystallogr. D Biol. Crystallogr. 53, 240-255 (1997).

Supplementary Information is linked to the online version of the paper at www.nature.com/nature.

Acknowledgements We thank A. Cheung and D. Kostrewa for help with crystallography, and W. T. McAllister and M. Anikin for the critical reading of the manuscript. We acknowledge the crystallization facility at the Max Planck Institute of Biochemistry, Martinsried. Part of this work was performed at the Swiss Light Source at the Paul Scherrer Institut, Villigen, Switzerland. Use of the Advanced Photon Source at Argonne National Laboratory was supported by the US Department of Energy, Office of Science, Office of Basic Energy Sciences, under contract no. DE-AC02-06CH11357. Use of the Lilly Research Laboratories Collaborative Access Team (LRL-CAT) beamline at Sector 31 of the Advanced Photon Source was provided by Eli Lilly Company, which operates the facility. P.C. was supported by the Deutsche Forschungsgemeinschaft, SFB646, TR5, FOR1068, the Nanosystems Initiative Munich NIM, an Advanced Investigator Grant from the European Research Council ERC, and the Jung-Stiftung. D.T. was supported by the UMDNJ Foundation, grant no. PC25-11

Author Contributions M.S. and D.L. cloned mtRNAP variants. M.S., D.L., D.T. and Y.I.M. performed RNAP purification and biochemical assays. R.R. and D.T. prepared the crystals. R.R. performed structure determination and modelling. P.C. and D.T. designed and supervised the project and prepared the manuscript.

Author Information Coordinates and structure factors of the human mtRNAP crysta structure are deposited with the Protein Data Bank under accession code 3SPA. Reprints and permissions information is available at www.nature.com/reprints. The authors declare no competing financial interests. Readers are welcome to comment on the online version of this article at www.nature.com/nature. Correspondence and requests for materials should be addressed to P.C. (cramer@lmb.uni-muenchen.de) or D.T. (d.temiakov@umdnj.edu). 